Int. J. Dev. Biol. 61: 439-450 (2017)

doi: $10.1387 / \mathrm{ijdb} .160277 \mathrm{sb}$

\title{
Segmentation gene expression patterns in Bactrocera dorsalis and related insects: regulation and shape of blastoderm and larval cuticle
}

\author{
WORRAMIN SUKSUWAN", XIAOLI CAI, LERTLUK NGERNSIRI* and STEFAN BAUMGARTNER* \\ Dept. of Experimental Medical Sciences, Lund University, Lund, Sweden
}

\begin{abstract}
The oriental fruit fly, Bactrocera dorsalis, is regarded as a severe pest of fruit production in Asia. Despite its economic importance, only limited information regarding the molecular and developmental biology of this insect is known to date. We provide a detailed analysis of $B$. dorsalis embryology, as well as the expression patterns of a number of segmentation genes known to act during patterning of Drosophila and compare these to the patterns of other insect families. An anterior shift of the expression of gap genes was detected when compared to Drosophila. This shift was largely restored during the step where the gap genes control expression of the pair-rule genes. We analyzed and compared the shapes of the embryos of insects of different families, $B$. dorsalis and the blow fly Lucilia sericata with that of the well-characterized Drosophila melanogaster. We found distinct shapes as well as differences in the ratios of the length of the anterior-posterior axis and the dorsal-ventral axis. These features were integrated into a profile of how the expression patterns of the gap gene Krüppel and the pair-rule gene even-skipped were observed along the A-P axis in three insects families. Since significant differences were observed, we discuss how Krüppel controls the even-skipped stripes. Furthermore, we discuss how the position and angles of the segmentation gene stripes differed from other insects. Finally, we analyzed the outcome of the expression patterns of the late acting segment polarity genes in relation to the anlagen of the naked-cuticle and denticle belt area of the $B$. dorsalis larva.
\end{abstract}

KEY WORDS: Bactrocera dorsalis, segmentation, Kruppel, even-skipped

The oriental fruit fly Bactrocera dorsalis (Hendel) (Diptera: Tephritidae) is a major cause of damage of fruit production in tropical and sub-tropical regions, often leading to a total failure of crop production (Vargas et al., 2015). It has in fact been classified as belonging to the most destructive category of insects (category A pests) (Vargas et al., 2015). B. dorsalis is widely prevalent in many countries in Asia, but also in the U.S. state of Hawaii. Since accidental introduction in the 1940's, B. dorsalis has established itself as a common pest. The insects have also occasionally been detected in the U. S. mainland, e. g. in California and Florida. Attempts have been made to eradicate the pest during four major infestations between 1960 and 1997. In Asia, eradication was not possible for monetary reasons initially. Consequently, damage on crop production was so severe that eradication programs were ultimately implemented to combat this insect.
Phylogenetically, Tephritidae is a family of fruit flies, located immediately adjacent of that of Drosophilidae (Yong et al., 2016). Both families belong to the sub-section Acalyptratae, and are referred to as "fruit flies", while the Drosophilidae are often referred to as "the common fruit fly". To make Tephritidae more distinct from Drosophilidae, they are often called "peacock flies". Both families maintain a similar body shape, however, Tephritidae are usually larger than Drosophilidae and are often more colorful with pictured wings. The third family that will be described below is Calliphoridae, a family that is phylogenetically even closer to Tephritidae than to Drosophilidae (Andere et al., 2016). Within the Calliphoridae, we will focus on the blow fly Lucilia sericata.

Abbreviations used in this paper: En, engrailed; Eve, even-skipped; Hb, hunchback; $\mathrm{Kr}$, Krüppel; otd, orthodenticle.

\footnotetext{
*Address correspondence to: Stefan Baumgartner. Dept. of Experimental Medical Sciences, Lund University, BMC D10, S-22184 Lund, Sweden. e-mail: Stefan.Baumgartner@med.lu.se

\#Present address: Department of Genetics, Faculty of Science, Kasetsart University, Bangkok, Thailand.
} 
Within the family of Tephritidae, several other insect members have been described to some detail such as Ceratitis capitata (the Mediterranean fruit fly) (Scolari et al., 2014) and B. oleae (the olive fly; Mavragani-Tsipidou, 2002). Common to this family is that all three fly species represent the most destructive of fruit pests. Ceratitis capitata, in particular, because of its wide distribution over the world, its ability to tolerate cooler climates better than most other species of tropical fruit flies and its wide range of hosts is ranked first among economically important fruit fly species.

The body of the adult $B$. dorsalis is about $8 \mathrm{~mm}$ in length, with a wingspan of about $7 \mathrm{~mm}$. Its body color is quite variable: the thorax is mainly dark brown with yellow stripes, while the abdomen is light brown with two black stripes perpendicular to the anteriorposterior (A-P) axis, and a black stripe along the midline. More than 150 kinds of fruits and vegetables are known to be attacked by $B$. dorsalis. Mango, papaya and avocado are the preferred food sources (Leblanc et al., 2012).

Embryogenesis and the formation of segments in insects follows two modes: in short-germband development, segments are added sequentially by adding them to the posterior of a growing embryo (reviewed by Davis and Patel, 2002). The blastoderm embryo occupies only a small fraction of the egg (the remainder consists of yolk and extra-embryonic tissue). Anterior segments are already determined during the blastoderm stage, while posterior segments are added only after gastrulation. In contrast, in insects using long-germband development (where $B$. dorsalis belongs to), the body axis is established already during oogenesis and segments are formed through subdividing the embryo into equally-sized subdomains (Davis and Patel, 2002). Most long-germband embryos take up a large proportion of the egg, and segments are already determined before gastrulation begins. Notissue growth is involved in this process. The morphological formation of segments occurs much later in development; segmental boundaries are visible only at the extended germband stage.

In Drosophila, the process of segmentation was described in detail and revealed that at the molecular level, a hierarchy of segmentation genes was crucial for setting up the anterior-posterior axis (Peel et al., 2005). Since segmentation is the common denominator of all insects, attempts have been made to integrate segmentation gene expression patterns of different insects into gene network models to detect common modes of regulation and to explain differences that were observed. The best defined gene regulation model is the gap-gene network model analyzed in dipteran insects (Crombach et al., 2016, Jaeger, 2011) which states that early activation and placement of gap gene expression domains show significant quantitative differences, yet the final patterning output of the system, i. e. the expression of pair-rule genes, is essentially identical in the species investigated, referred to as " system drift" (Crombach et al., 2016). However, information is still limited in regard to comparative gene expression sets in insect families which would allow to test this network model.

An important question is how do the gap genes regulate the pair-rules genes and is this regulation conserved between insect families? For the former question, some progress was made, mainly in Drosophila, due to the availability of mutants and the possibility of manipulating this insect. Major focus was on the gap genes Krüppel, hunchback, giant and knirps, and how these genes regulated the different stripes of the pair-rule gene even-skipped (Goto et al., 1989; Harding et al., 1989; Stanojevic et al., 1991; Small et al., 1996). These reports extended our understanding how the interplay of gap genes control the different even-skipped stripes, and defined an initial set of transcriptional activators and repressors for each of the even-skipped stripe enhancers. For example, even-skipped stripe 2 appeared because it was activated by the bicoid and hunchback gradients, but repressed by giant anteriorly to its margin and by Krüppel posteriorly to its margin, giving rise to a 4-cell-wide expression of even-skipped stripe 2 (Small et al., 1996). To further understanding as to whether or not even-skipped enhancers were conserved between insects families, even-skipped enhancers were identified in scavenger flies, Sepsidae (Hare et al., 2008), but the sequence identity of the enhancers to those of Drosophila was unexpectedly low. This report demonstrated that the identification of regulatory sequence by simple sequence comparison in other insects would not be successful, unless yetunestablished functional studies would be performed as well. To circumvent this issue, in silico approaches were developed to model the transcription of Drosophila even-skipped stripe 2 (Ilsley et al., 2013). To date, the only method to reliably detect similarities or differences of how gap genes activate the pair-rule genes in different insect families is to compare the relative positions of gap genes and pair-rule gene stripes along the AP-axis and to determine whether or not a particular gap gene can exert control on a particular pair-rule gene stripe.

As far as the analyses of expression patterns of segmentation genes in Tephritidae is concerned, only limited information is available. One report identified plasticity in expression with orthodenticle (otd), an early segmentation gene in the medfly, Ceratitis capitata and the caribfly, Anastrepha suspensa (Schetelig et al., 2008), suggesting that changes in otd expression can occur even in closely related taxa. Another report showed that the maternal gene nanos of the medfly was expressed in a similar pattern as Drosophila forming a posterior mRNA gradient, but not identifying plasticity between the two families (Ogaugwu and Wimmer, 2013).

Despite its significant impact on the world-wide fruit market, little is known about the molecular and developmental biology of $B$. dorsalis. Recently, some progress was made in developing genomic resources for this species after the complete sequencing of the genome and some detailed analysis (Calla and Geib, 2015; Geib et al., 2014; Sim et al., 2015). In addition, RNA sequencing of the transcriptome of genes involved in sexual maturation and mating led to the identification of important genes involved in the sexual development of the $B$. dorsalis female, which consequently may help to develop sterility programs (Zheng et al., 2016). Concurrently, a draft sequence of a relative of $B$. dorsalis, B. tryoni, was made available in 2014 (Gilchrist et al., 2014). However, only limited information on the embryology and developmental biology of Bactrocera is available, with the exception of a superficial description of the biology of $B$. tau (Singh, 2010) and that of another Tephritid, B. tryoni (Anderson, 1964).

To supplement the information currently available in describing $B$. dorsalis embryology, we have characterized its embryonic development in detail. We describe the embryonic development of this species, present a panel of expression patterns of early segmentation genes and compare these to the know expression patterns of other established model insects. To address the question whether or not the control of the pair-rule genes by the gap genes is conserved between three insect families, we superimposed the expression patterns of the gap gene Krüppel to those of the pair- 
rule gene even-skipped and draw conclusions how Krüppel can control the even-skipped stripes in the different insect families. We also noted that the angle and behavior of the even-skipped stripes are distinct from those of previously characterized insects with respect to the A-P axis. Furthermore, we show that the shape of the $B$. dorsalis egg and the behavior of the nuclei during the blastoderm stage is considerably different from that of previously characterized insects. Lastly, we analyzed the outcome of the expression patterns of the late acting segment polarity genes in relation to the anlagen of the naked-cuticle and denticle belt area of the $B$. dorsalis larva.

\section{Results}

\section{Embryogenesis of $B$. dorsalis}

To date, only sparse information of $B$. dorsalis embryogenesis and postembryonic development exists. Some information exist for two closely-related species, B. tau (Singh, 2010) and B. tryoni (Anderson, 1964), respectively. For this reason, we have analyzed and summarized relevant stages of the $B$. dorsalis life cycle in Fig. 1 . We refer to the nomenclature of the stages of Drosophila embryogenesis as previously described (Campos-Ortega and Hartenstein, 1985 ) and also to those of the blow fly Lucilia sericata (Mellenthin et al., 2006; Blechert et al., 2011). Embryonic development of $B$. dorsalis proceeds as a long germband insect (Davis and Patel, 2002). As in most insects, the egg is surrounded by a vitelline membrane and a chorion, however, in contrast to Drosophila, no dorsal appendages are observed. Once the chorion is removed, an egg is revealed which is about $0.8 \mathrm{~mm}$ long and $0.3 \mathrm{~mm}$ in diameter (Fig. 1). The ratio of the length to width, however, is quite different from that of Drosophila rendering the egg prone to pressure and physical manipulations. The ventral side as well as the dorsal side are usually curved, a feature not found in Drosophila. The posterior tip is often pointed (Fig. 1D) giving the egg a distinct form, compared to those of Drosophila or Lucilia (Fig. 7B).

After fertilization, the first nuclear cleavages are very similar to those of Drosophila where the nuclei divide in the interior of the yolk (Fig. $1 \mathrm{~A}, \mathrm{~B}$ ) until they migrate to the periphery to form a syncytial blastoderm (Fig. 1B). In contrast to Drosophila where the pole cells (the future germ cells) are formed at nuclear cycle (nc) 10 at the posterior pole, the pole cells are only formed at nc 12 in $B$. dorsalis (Fig. 1C). As in Drosophila, after nc 10, four more nuclear cycles follow until the stage of cellular blastoderm is reached (Fig. $1 \mathrm{C}, \mathrm{D})$. Interestingly, during nc 14 , no elongation of nuclei occurs as is observed in Drosophila, hence nuclei remain rather round during this nc (Fig. 1D). At about 6 hours after egg deposition, the first signs of gastrulation become obvious (Fig. 1E). However, the shape of the cells still does not change and they remain round. Anteriorly, a prominent circumferential furrow is observed which corresponds to the cephalic furrow in Drosophila. Ventrally, the mesoderm starts to invaginate (Fig. 1E). On the lateral side, up to 4 folds are observed. This is due to the fact that the radius of the

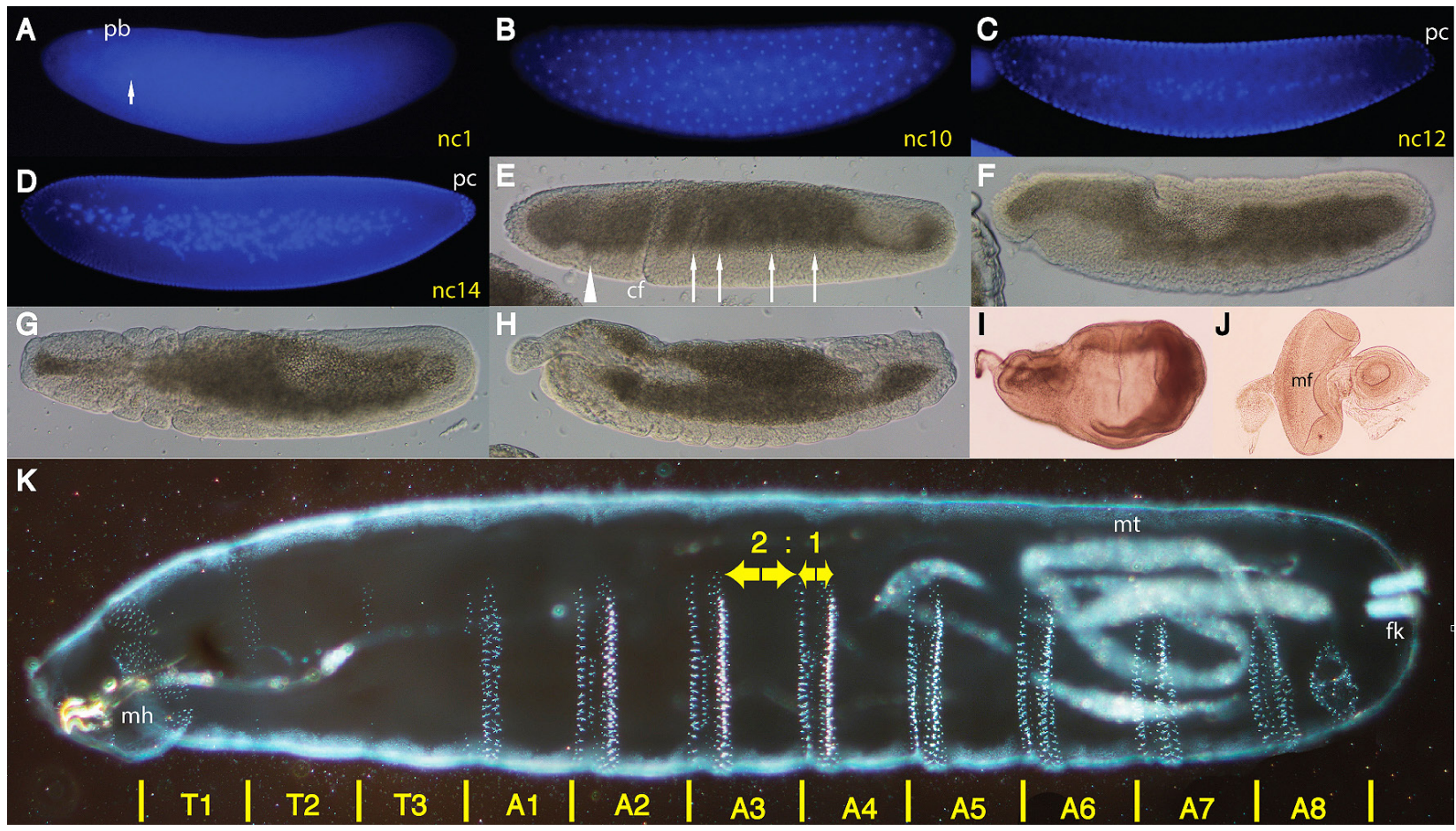

Fig. 1. Embryogenesis and imaginal discs of $\boldsymbol{B}$. dorsalis. (A-K) All embryos are oriented anterior to the left and dorsal side up, unless otherwise noted. (A-D) DAPI staining, nuclear cycles (nc) are indicated in yellow. (A) Nuclear cycle (nc) 1 embryo staining showing the polar body (pb) and the zygotic nucleus (arrow). (B) Nc 10 embryo. Note absence of pole cells. (C) Nc 12 embryo. Note the presence of the pole cells (pc) as a small number of cell at the posterior tip. (D) Nc 14 showing the pole cells (pc).(E-J) Bright field microscopy. (E) Gastrulating embryo showing the cephalic furrow (cf) and up to 4 posterior transverse fold (arrows). Anteriorly, an anterior fold is observed (arrowhead). (F) Extended germband embryo. (G) Embryo during germband retraction. (H) Germband retracted embryo. (I) $3^{r d}$ instar wing disc. (J) $3^{\text {rd }}$ instar eye-antennal disc showing the morphogenetic furrow (mf). (K) Dark field picture of a $1^{\text {st }}$ instar larva with annotation of the segments and segmental borders (in yellow). The ratio of the distance of naked cuticle versus denticle belts is indicated above A3. Abbreviations: $m$ h, mouth hook; mt, malphigian tubules; fk, filzkörper. 
curve leading to germband extension is smaller in Bactrocerathan in Drosophila with respect to the dimension of the body axes. 10 hours into development, the germband is fully extended (Fig. 1F), and its shape appears quite similar to that of Drosophila or Lucilia. At approximately 14 hours of development, the germband starts to retract (Fig. 1G) taking about 10 hours to complete retraction (Fig. $1 \mathrm{H})$. The following morphogenetic movements such as head involution or dorsal closure proceed as they do in Drosophila, however, the speed by which they progress is considerably slower, as noted by (Vargas, 2000; Anderson, 1964). Only after approximately 48 hours at $25^{\circ} \mathrm{C}$, the larva hatches (Fig. $1 \mathrm{~K}$ ) showing an identical number of segments compared to Drosophila or Lucilia, a head, 3 thoracic segments, 8 abdominal segments and a tail (Jurgens, 1987; Martinez Arias, 1993; Mellenthin et al., 2006). The patterns of the denticle bands are distinct from that of Drosophila (Moline et al., 1999) or that of Lucilia (Mellenthin et al., 2006), showing a gap between weaker anterior rows and stronger posterior rows (Fig. 1K), the latter caused by thicker individual denticle hairs. The ratio of the distance of naked cuticle to that of the denticle bands was about 2:1, a feature distinct from other insects (Fig. $1 \mathrm{~K}$; Mellenthin et al., 2006).

$B$. dorsalis undergoes 3 larval stages, as does Drosophila and Lucilia. The larval stages last about 8 days at $24^{\circ} \mathrm{C}$ (Vargas, 2000). These values are also larger than those of Drosophila or Lucilia. During the end of the $3^{\text {rd }}$ instar larval stage, B. dorsalis larvae show a peculiar behavior inherent to most Tephritidae: the larvae bend their body by arresting their muscles and releasing the tension which enables them to jump as far as 50 centimeters and as high as 30 centimeters. This behavior is interpreted as an attempt to leave the habitat and to find a secluded place for pupation. The pupal stage takes about 12 days at $24^{\circ} \mathrm{C}$ (Vargas, 2000). Hence, the complete life cycle of $B$. dorsalis can take as long as 19 days at $24^{\circ}$ (Vargas, 2000), 40-50\% longer than in Drosophila, mostly due to the prolonged larval and pupal stage.

To determine if $B$. dorsalis utilizes imaginal discs to form the precursors for the adult structures, $3^{\text {rd }}$ instar larvae were dissected to look for conspicuous discs such as wing and an eye-antennal discs to investigate if they resemble those of Drosophila (Fig. 1I, J). A wing disc is similar in shape to that of Drosophila (Fig. 1I) and so is an eye-antennal disc (Fig. 1J). Photoreceptor cells follow a similar developmental fate as in Drosophila or Lucilia, as revealed by the existence of a morphogenetic furrow (Fig. 1J). Altogether, this data demonstrates that imaginal disc development of $B$. dorsalis and probably that of most Tephritidae resembles strongly that of Drosophilidae or Calliphoridae.

\section{B. dorsalis segmentation genes}

In order to analyze Bactrocera segmentation gene expression, we first screened a database (Geib et al., 2014) for the presence of orthologous segmentation genes of the prime model system Drosophila melanogaster. In Drosophila, the process of segmentation was described in detail showing that a hierarchy of segmentation genes is crucial for setting up the anterior-posterior axis (Peel et al., 2005). In Bactrocera, not all genes of this hierarchy were found (Geib et al., 2014). For example, the maternal gene bicoid (bcd), shown to be a feature of higher Diptera only, is not present in the Bactrocera genome. In Drosophila, bicoid is expressed in a protein gradient along the A-P axis (Driever and Nüsslein-Volhard, 1988), preceded by the formation of a mRNA gradient (Frigerio et al., 1986; Spirov et al., 2009; Fahmy et al., 2014). The protein gradient serves as morphogen gradient to pattern the anterior-posterior axis. In insects where bicoid is lacking, it was proposed that two genes described in Drosophila as gap genes, the hunchback $(h b)$ and the orthodenticle (otd) act cooperatively to pattern the anteriorposterior axis, instead (Peel et al., 2005; Schetelig et al., 2008).

With this in mind, we have analyzed the expression patterns of important Bactroceramembers of the segmentation gene hierarchy (Nüsslein-Volhard and Wieschaus, 1980). Of the class of genes at the top of the hierarchy, we analyzed the $h b$ and otd gene, followed by a member of the gap gene class, the Krüppel $(K r)$ gene. The pair-rule gene class is represented by the even-skipped(eve) gene, while the segment polarity gene class by the engrailed (en) gene.

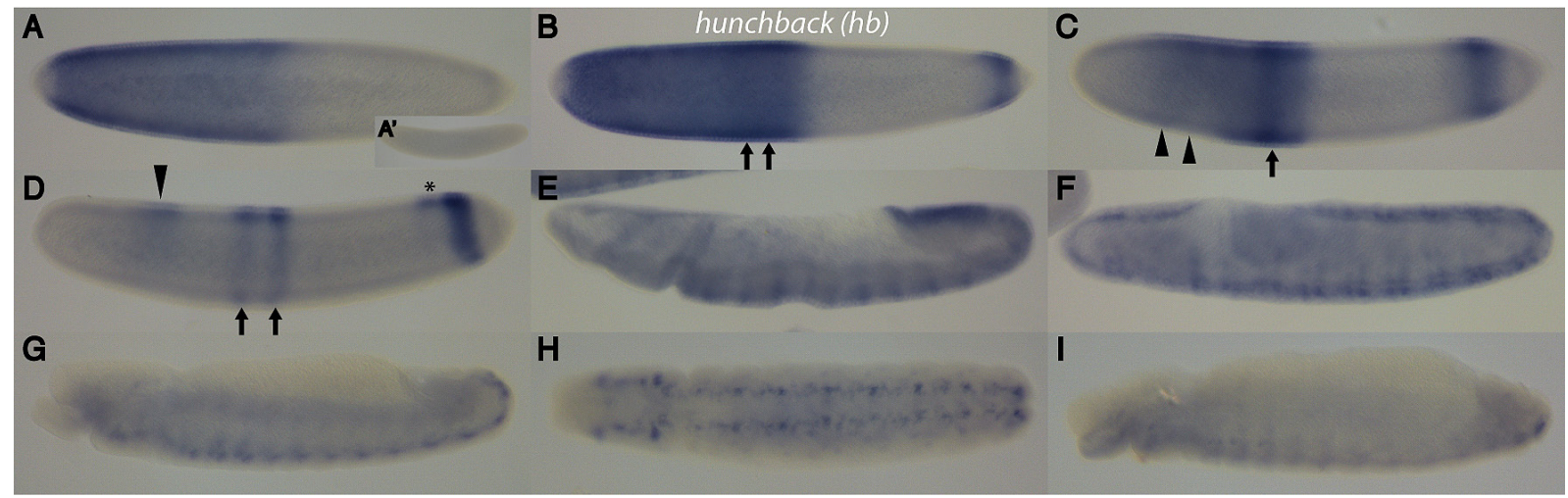

Fig. 2. Embryonic expression of B. dorsalis hunchback (hb). (A-I) All embryos are oriented anterior to the left and dorsal side up, unless otherwise noted. (A) Nc 13 embryo, hb expression is in a broad anterior domain. (A') Early cleavage stage embryo, no maternal hb expression is detected. (B) Early nc 14 embryo, an additional posterior band emerges. In the anterior broad band, the posterior end increases transcription (arrows). (C) Late nc 14 embryo, the anterior end of the broad domain looses transcripts (arrowheads). In the posterior part, a distinct band becomes visible (arrow). (D) Early gastrulation embryo, the broad anterior band has resolved into a dorsal band (arrowhead) and two adjacent bands (arrows), while the posterior band shows a dorsally-expressed spot (asterisk) and the residual strong posterior band. (E) Germband extension, a repetitive pattern of ectodermal stripes emerges. (F) Extended germband, transcripts are in a segment-specific pattern in specific neuroblasts. (G,H) Germband retracted embryo, lateral (G) and ventral (H) view, respectively, a repetitive segmental pattern in neuroblasts is observed. (I) Late embryogenesis, transcripts persist mainly in the ventral chord. 


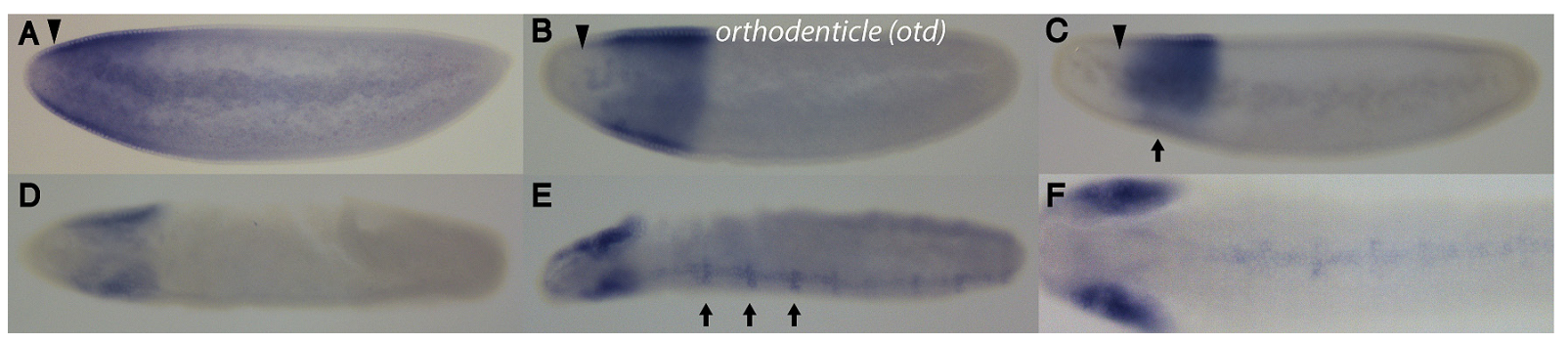

Fig. 3. Embryonic expression of B. dorsalis orthodenticle (otd). (A-F) All embryos are oriented anterior to the left and dorsal side up, unless otherwise noted. (A) Early nc 14 embryo, otd is expressed in a broad domain, leaving the anterior-most tip free (arrowhead). (B) Late nc 14 embryo, a large area of the tip does no longer show hb transcripts (arrowhead). (C) Early gastrula embryo, anteriorly, transcripts disappear (arrowhead), as well as in the ventral-most region (arrow). (D) Embryo during germband extension, hb transcripts are detected in two large lateral patches. (E) Extended germband embryo, the anterior patches remain, while midline cells show transcripts with a segmental pattern (arrows). (F) Extended germband, ventral view, the lateral patches remain as well as expression in midline cells.

\section{Expression of hunchback (hb)}

Expression of Bactrocera $h b$ commenced at nuclear cycle (nc) 13 where a broad domain extending was detected from about $5 \%$ to $55 \%$ egg length (EL, where the anterior tip is defined as $0 \%$ and the posterior tip as 100\%; Fig. 2A). In Drosophila, this expression is similar, although the anterior domain included the whole anterior tip (Bender et al., 1988; Tautz and Pfeifle, 1989), FlyBase (Attrill et al., 2016). In contrast to Drosophila, however, no ubiquitous maternal $h b$ contribution in Bactrocera was detected during the early nuclear stages (Fig. 2A'). At early nc 14, the posterior end of the broad $h b$ domain showed increased levels of transcription (Fig. 2B, arrow), while at the posterior end a posterior stripe appeared. During later stages of nc 14, the anterior end of the broad domain showed decreased levels of $h b$ (Fig. 2C, arrowheads) and the posterior band increased in intensity. At early gastrula (Fig. 2D), expression of the anterior domain almost completely ceased, with the exception of two distinct stripes in the middle (Fig. 2D, arrows) and an anterior dorsal patch (Fig. 2D, arrowhead). The posterior band remained strong with the anterior end showing expression only dorsally (Fig. 2D, asterisk). In Drosophila, a similar pattern was observed (Attrill et al., 2016), however, only one stripe was found in the middle and the stripe was shown shifted to the anterior, in comparison to Bactrocera. During germband extension (Fig. 2E), a repetitive pattern of $h b$ stripes evolved, with the posterior-most expression showing strongest expression. At extended germband (Fig. 2F), strong expression was observed in neuroblasts in a segmented pattern. During germband retraction (Fig. $2 \mathrm{G}, \mathrm{H}$ ), $h b$ expression was observed in neuroblasts. During late embryogenesis (Fig. 2l), many neurons in the ventral nerve chord showed strong $h b$ expression.

\section{Expression of orthodenticle (otd)}

Expression of otd initiated as a broad circumferential anterior domain at early nc 14, ranging from $2 \%$ to $34 \%$ EL (Fig. $3 \mathrm{~A}$ ) and leaving the tip free (Fig, $3 A$, arrowhead), thus clearly later than $B$. dorsalis $h b$ (Fig. 2A) and making otd a possible target of regulation by $h b$. During nc 14 (Fig. 3B), the anterior edge shifted to about $11 \%$ EL (Fig. 3B, arrowhead), while the posterior edge remained

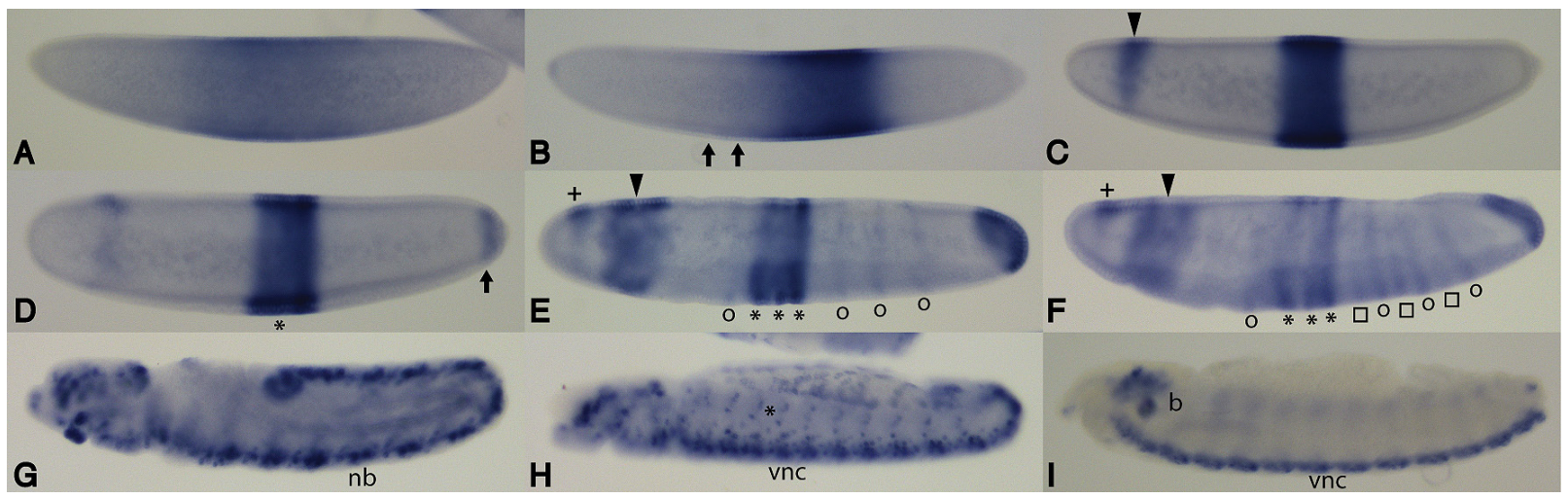

Fig. 4. Embryonic expression of B. dorsalis Krüppel (Kr). (A-I) All embryos are oriented anterior to the left and dorsal side up, unless otherwise noted. (A) Nc 13 embryo, a broad Kr band is observed. (B) Early nc 14 embryo, Kr transcripts disappear at the anterior end of the broad band (arrows). (C) Late nc 14 embryo, a central domain has emerged and anteriorly, a dorsal "cap" appears (arrowhead). (D) Early gastrula embryo, a posterior band appears (arrow). The middle band begins to split up (asterisk). (E) Embryo at beginning of germband extension, the band in the middle shows clear splitting into 3 bands (asterisk) and 3 new weak bands appear posteriorly and one anteriorly to the central band (open circles). Anteriorly, the dorsal cap becomes stronger (arrowhead). Even more anteriorly, a new cap band appears (+). (F) Embryo during germband extension, slightly older than that in (E), posterior to the middle band which remains split as 3 separate bands, a series of new bands (squares) appear posteriorly which alternate with those marked by open circles making a total of 10 consecutive bands with alternate intensities. (G) Extended germband embryo, strong transcription is observed in neuroblasts ( $\mathrm{nb})$. (H) Retracted germband embryo, $\mathrm{Kr}$ transcripts are seen in the ventral nerve cord (vnc) and in certain muscle precursor cells (asterisk). (I) Embryo shortly before hatching, transcripts are detected in the ventral nerve chord (vnc) and in the brain (b). 
constant. During early gastrula (Fig. 3C), transcripts disappeared further from the anterior edge (Fig. 3C, arrowhead) and comprised a band from $17-34 \%$ EL. At the ventral side, the presumptive mesodermal anlagen was devoid of any transcripts (Fig. 3C, arrow), similar to that in Drosophila (Finkelstein et al., 1990; Attrill et al., 2016). During germband extension (Fig. 3D), the anterior domain remained. At extended germband (Fig. $3 \mathrm{E}, \mathrm{F}$ ), a two-cell wide stripe with lateral extensions emerged (Fig. 3E, arrows). These cells corresponds to the midline cells that marked the outermost cells of the invaginating mesoderm. A similar expression in midline cells was also observed in Drosophila (Finkelstein and Perrimon, 1990; Attrill et al., 2016). However, while transcription of otd continues in Drosophila, otd transcripts in B. dorsalis ceased after the extended germband stage. Contrary to our expectation, we noted that in two other Tephritidae, the medfly Ceratitis capitata and the caribfly Anastrepha suspensa, maternal otd expression was observed (Schetelig et al., 2008). Moreover, medfly otd showed a wide band of expression at the early blastoderm expression, and only at cellular blastoderm, all three Tephritidae otd patterns converged to an identical broad anterior band of about $17-34 \%$ EL. Therefore, there is considerable plasticity of otd gene regulation between close relatives within the same family, also noted by (Schetelig et al., 2008).

\section{Expression of Krüppel (Kr)}

$K r$ expression in $B$. dorsalis started with a broad band in the middle of the embryo from about 30\% EL to $75 \%$ EL with tapered expression on either side at nc 13 (Fig. 4A). Its occurrence paralleled that of $h b$ (Fig. 2A). At early nc 14, the anterior-most part of the band diminished (Fig. 4B, arrows) and transcripts were observed in a band from $45 \%$ to $70 \%$ with a sharp posterior boundary. At late nc 14 (Fig. 4C), a band from $45 \%$ to $60 \%$ with sharp anterior and posterior boundaries emerged, while anteriorly, a new band appeared whose width tapered off on the ventral side (Fig. 4C, arrowhead). At early gastrula stage (Fig. 4D), a posterior band appeared and the broad middle band began to split (Fig. 4D, asterisk). Slightly later, at the anterior end, another band appeared (Fig. 4E, marked with + ), while the initial anterior bands broadened and segregated.

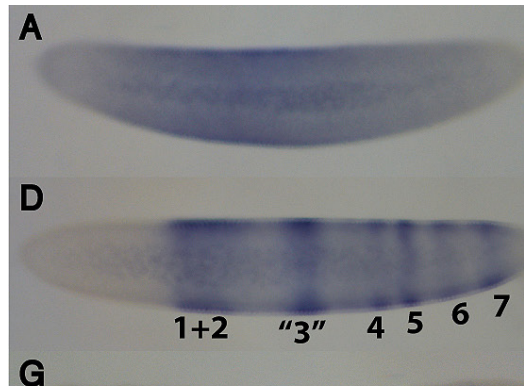

G

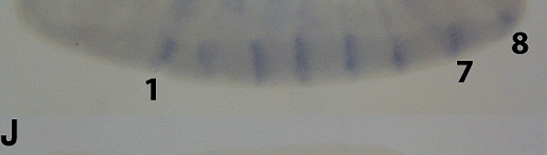

$J$

$\operatorname{tr}$

nb

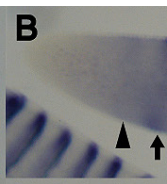

E

\section{$\uparrow$}

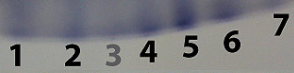

H

8

K
$\Lambda$

$\Lambda$

8

even-skipped (eve)

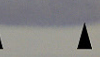

8

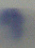

c

$\mathbf{F}$

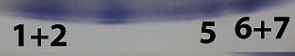

I
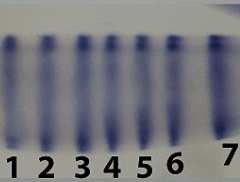

$\operatorname{tr}$

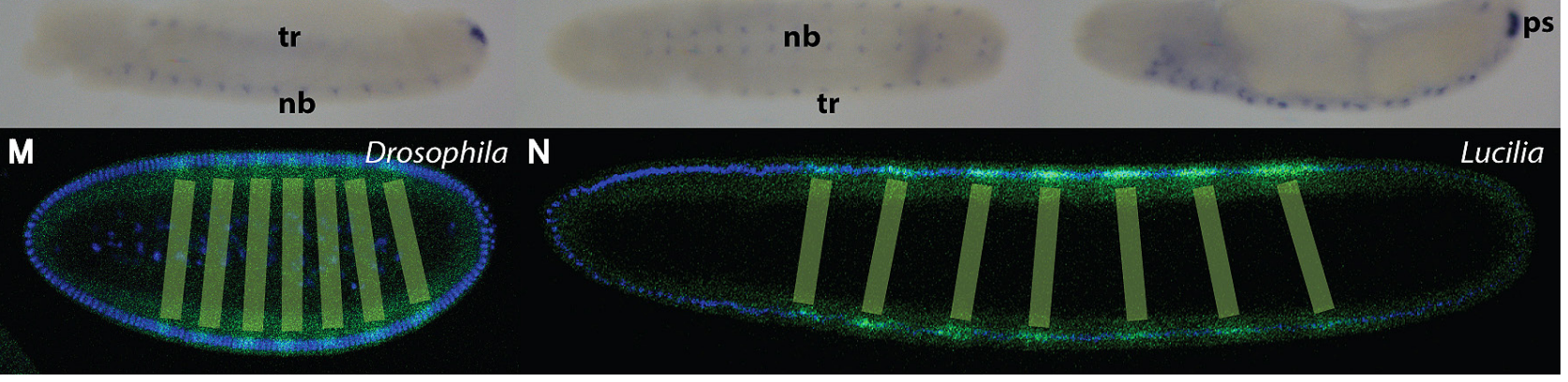

Fig. 5. Embryonic expression of B. dorsalis even-skipped (eve). (A-N) All embryos are oriented anterior to the left and dorsal side up, unless otherwise noted. (A) Early nc 14 embryo, eve is expressed in a broad central domain with diffuse ends. (B) Early nc 14 embryo slightly later than in (A), an anterior band (arrow) appears, along with further weak bands (arrowheads). (C) Mid nc 14 embryo, the weak bands become stronger and anteriorly, another broad band appears, forming bands 1, 2, 5, 6 and 7. (D) Mid nc 14, slightly older than (C), 6 bands with different widths and intensities evolve. Bands 4-7 have final width and positions, bands 1-3 are still not determined. Numbering of stripes according to the final 7 stripe-pattern in (F). (E) Nc 14 embryo close to cellularization, all bands are established, band 3 is considerably weaker. (F) Early gastrula embryo, 7 bands with equal intensity have evolved. (G) Embryo during germband extension, posterior to band 7, band 8 appears. Bands 1-7 become weaker. (H) Extended germband embryo, only the posterior-most band 8 remains. (I) Embryo at beginning of germband retraction, the posterior band is still visible. Single cells, presumably in tracheal precursors (tr), start to express eve. (J) Germband retracted embryo, a repetitive pattern in certain neuroblasts ( $n b)$ and tracheal cells (tr) emerges. (K) Ventral of a similar staged embryo as in (J), expression is conspicuous in neuroblasts and tracheal cells. (L) Late embryogenesis, eve expression is in the ventral nerve chord in neuroblasts and the posterior spiracle (ps). (M) Confocal picture of a late nc 14 of a D. melanogaster embryo, Eve protein expression is in green, along with DAPI (blue) to reveal the nuclei. The angle of the 7 stripes with respect to the A-P axis is indicated in shaded green. (N) Confocal picture of a late nc $14 \mathrm{~L}$. sericata embryo, Eve protein expression is in green, along with DAPI (blue) to reveal the nuclei. The angle of the 7 stripes with respect to the A-P axis is indicated in shaded green. 
The band in the middle divided into 3 bands (Fig. 4E, marked by asterisk). Posterior to the middle bands, 3 weak new stripes appeared and anteriorly one additional band was detected (Fig. 4E, marked by "o"). During germband extension (Fig. 4F), a faint set of stripes (Fig. 4F, marked by squares) appeared between the 3 posterior bands marked with "o". Hence, at this stage, a row of 10 distinct bands with different intensities emerged, subdividing the region at $40 \%$ to $80 \%$ EL of the embryo into a striped pattern, reminiscent of segment polarity gene expression (Fig. 6). During extended germband (Fig. 4G), all segments exhibited strong $\mathrm{Kr}$ expression, mainly in specific neuroblasts $(\mathrm{nb})$. During germband retraction (Fig. $4 \mathrm{H}$ ), strong $K r$ expression was in neuroblasts of the ventral nerve cord (vnc) and in certain muscle precursor cells (asterisk). During late embryogenesis (Fig. 4I), Kr was strongly expressed in the brain (b) and the ventral nerve chord (vnc).

Krexpression has been analyzed in a variety of insects: Drosophila melanogaster(Jaeckle etal., 1986; Gaul etal., 1987), Musca domestica (Sommer and Tautz, 1991), Clogmia albipunctata (Rohr et al., 1999; Garcia-Solache et al., 2010), Oncopeltus fasciatus (Liu and Kaufman, 2004), Tribolium castaneum(Bucher and Klingler, 2004), Nasonia vtiripennis (Olesnicky et al., 2006), Episyrphus balteatus (Lemke et al., 2010), Apis mellifera (Wilson et al., 2010), Lucilia sericata (Blechert et al., 2011) and Bombyx mori (Nakao, 2015). In comparison to the aforementioned insects, the evolution of the complex $B$. dorsalis Krexpression pattern at blastoderm and early gastrulation resembled that of $L$. sericata $K r$ (Blechert et al., 2011), as similar banding patterns were seen in the anterior, middle (Fig. 4D) and biphasic onset of the posterior weak stripes (Fig. 4E, F).

\section{Expression of even-skipped (eve)}

Expression of eve began at early nc 14 (Fig. 5A) revealing a broad band with diffuse ends. At early nc 14 (Fig. 5B), an anterior strong band was visible (Fig. 5B, arrow), along with some minor bands (Fig. 5B, arrowheads). At mid nc 14 (Fig. 5C), a more defined banding pattern appeared with broad anterior and posterior bands, respectively. In the middle, a strong but transitional band appeared. Thereafter, but still during mid nc 14 (Fig. 5D), the posterior broad band split up, and a new band, band 4, appeared. Anteriorly, the broad band (band $1+2$ ) remained together, while the identity of the middle band (referred to as " 3 ") still remained obscured. Close to cellularization (Fig. 5E), all bands resolved, with the exception of band 3 which was still weak. At early gastrulation (Fig. 5F), 7 bands with a width of 3 cells evolved without regular spacing. Notably, band 7 was more posteriorly-located. During germband extension (Fig. 5G), a new band 8 appeared which was the only one to remain at extended germband (Fig. $5 \mathrm{H}$ ). During germband retraction (Fig. $5 \mathrm{I})$, a segmental pattern at certain tracheal cells emerged while the posterior band remained defined. At retracted germband (Fig. 5 $\mathrm{J}, \mathrm{K})$, the pattern remained unchanged in the tracheal cells, while neuroblasts showed eve transcripts in a repetitive pattern. During late embryogenesis (Fig. 5L), eve transcription remained high in neuroblasts and the posterior spiracles.

To compare the relative position along the A-P axis and the spatial geometry of the eve stripes in some related phyla, $D$. melanogaster and $L$. sericata embryos were stained with monoclonal antibody 2B8, known to detect the Eve protein in distant insect families. While 2B8 did not detect Eve in B. dorsalis (data not shown), it revealed 7 stripes in $D$. melanogaster (Fig. $5 \mathrm{M}$ ) and $L$. sericata (Fig. $5 \mathrm{~N}$ ). Interestingly, in comparison to $B$. dorsalis (Fig. $5 \mathrm{~F}$ ) where the stripes were perpendicular to the A-P axis, the angles of the anterior-most and posterior-most stripes were tilted towards the dorsal side, respectively (Fig. $5 \mathrm{M}, \mathrm{N}$ ). While this could easily be explained in the case of $D$. melanogaster by the fact that the dorsal side was rather straight and the ventral side rounded (Fig.

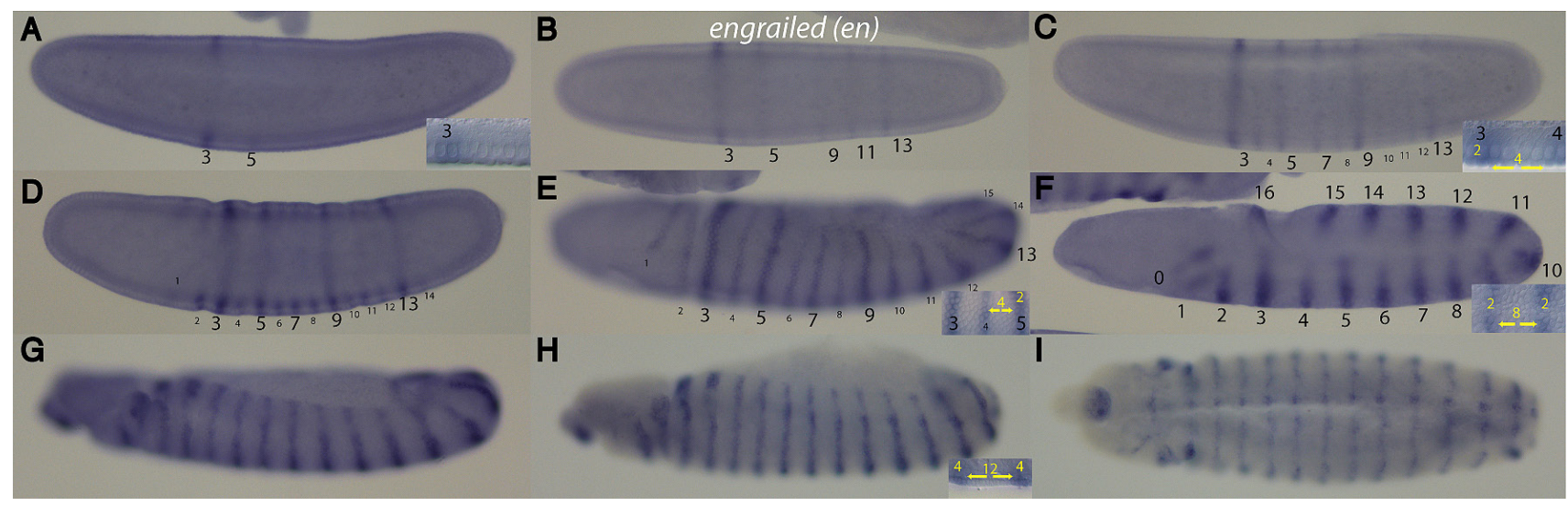

Fig. 6. Embryonic expression of B. dorsalis engrailed (en). (A-I) All embryos are oriented anterior to the left and dorsal side up, unless otherwise noted. Numbering of stripes is according to the final 17 stripe-pattern in (F). (A) Late nc 14 embryo, en transcripts are detected in a 2-cell wide stripe 3 and posteriorly a weaker stripe 5. Insert shows width of 2 cells in stripe 3. (B) Nc14, cellular blastoderm embryo, posterior to stripe 5, stripes 9, 11 and 13 appear. Stripe 7 is delayed. (C) Early gastrula embryo, odd-numbered stripes 3-13 become established. In between, even-numbered stripes 4-12 appear with the exception of stripe 10 which is delayed. Insert shows the width of stripe 3 of 2 cells, as well as the interstripe section of 4 cells (indicated by arrows) and stripe 4. (D) Early gastrula embryo, slightly later than in (C), stripes 1, 2 and 14 appear. The stripe intensity is not equal, the odd-numbered ones are stronger than the even-numbered ones. (E) Germband extension embryo, posterior to stripe 14, stripe 15 appears. The intensity of the stripes is still not equal. Insert shows the area of stripe 3 to 5 with width of 2 cells for the stripes and 4 cells for the interstripe region. (F) Extended germband embryo, 16 stripes with equal intensity have evolved. Insert shows the area of stripe 4 to 5 with width of 2 cells for the stripes and 8 cells for the interstripe region. (G) Embryo during germband retraction, the number of stripes remains identical. (H,I) Germband retracted embryos, lateral and ventral view, respectively. Insert in $\mathbf{( H )}$ shows the area of stripe 8 to 9 with a width of 4 cells for the stripes and 12 cells for the interstripe region. All stripes are close to the posterior border of each segment. 
$5 \mathrm{M})$, difficulties arose to explain the observation in the case of $L$. sericata (Fig. $5 \mathrm{~N}$ ) which has a similar body shape and A-P/D-V axis ratio as $B$. dorsalis (Fig. $5 \mathrm{~F}$ ).

\section{Expression of engrailed (en)}

$B$. dorsalis en expression was first detected at late nc 14 in a two-cell wide stripe and a posterior weaker stripe (Fig. 6A). The stripes correspond to stripes 3 and 5 and were within the position of the first appearance of the striped expression of eve (insert of Fig. 6A). Shortly thereafter, at cellularization (Fig. 6B), stripes 9, 11 and 13 emerged, comprising the first wave of en expression in odd-numbered bands. During early gastrula (Fig. 6C), the oddnumbered stripes were complemented by weaker even-numbered stripes in between, a pattern which progressed through germband extension (Fig. 6 D,E) with 2 cells expressing en followed by a gap of 4 cells, making a total of 6 cells/segment (insert in Fig. 6E). At extended germband, even- and odd-numbered stripes reached identical intensities (Fig. 6F). Anteriorly, stripes 0 and posteriorly stripes 15 and 16 became visible, making a total of 17 stripes. The width of the en bands was 2 cells and the interstripe region 8 cells (insert in Fig. 6F). Hence, one segment comprised of about 10 cells at this stage. During germband retraction (Fig. 6G), a striped pattern in the ectoderm in the posterior of each segment was visible, which persisted during the remaining stages of embryogenesis (Fig. $6 \mathrm{H}, \mathrm{I}$ ). The number of en-expressing cells increased to 4 cells and the interstripe region to 12 cells, making a total of 16 cells/segment (insert in Fig. 6H).

\section{Evo-Devo: comparative expression analysis reveals distinct interpretation of the gap signal at the pair-rule level}

Asurvey in the literature revealed that the expression of $K r$ was investigated in many insects, a comprehensive list is presented above. Hence, it serves as a prime marker to understand how gene expression correlates with the geometry and layout of the insect embryo. Of these aforementioned insects, only a handful were long germband insects: Drosophila melanogaster (Gaul et al., 1987), Musca domestica (Sommer and Tautz, 1991), Episyrphus balteatus (Lemke et al., 2010) and Lucilia sericata (Blechert et al., 2011). This collection of data nevertheless permitted the establishment of a map of their expression domains with respect to the A-P axis (Fig. 7A). This comparative map revealed that the majority of long germband insects showed $K r$ expressed in a band between 40-53/57\% $E L$, with $L$. sericata showing a distinct anterior shift (30-45\% EL, (Blechert et al., 2011) and B. dorsalis a marginal posterior shift (45-60\% EL).

Surprisingly, when eve banding patterns were compared (Fig. 7B), these considerable differences at the gap-gene level were almost completely restored: The anterior $L$. sericata eve bands were still more anterior compared to the "reference" bands of $D$. melanogaster and

B
$B$. dorsalis, however, the percentage of the difference compared to $K r$ diminished. Interestingly, the posterior-most bands were at similar levels, despite the difference of $12-15 \%$ at the posterior end of the $K r$ bands. We conclude that a compensation of the relative position of the segmental anlagen must have occurred during the stage from the gap gene to the pair-rule gene level which is more pronounced at the posterior part of the embryo.

To address the question if gap genes showed a conserved behavior of controlling the pair-rule genes between the three insect families, we superimposed the Kr expression domains to the eve stripes (Fig. 7B). We noted significant differences: in Drosophila, the anterior margin of the $K r$ band is congruent to the posterior margin of eve stripe 2, consistent with the notion that $K r$ demarcates the posterior end of eve stripe 2 (Small et al., 1996). In Lucilia, we noted the dramatic anterior shift of the $K r$ domain (Blechert et al., 2011), but when this domain was superimposed to that of eve, the anterior margin of the $K r$ band was congruent to the posterior margin of eve stripe 1, and not to that of stripe 2. In Bactrocera, however, the anterior margin of the $K r$ band was congruent to the posterior margin of eve stripe 2, as in Drosophila, suggesting a similar control. What was common to all three insect families was the fact that the $K r$ band straddled two adjacent eve stripes. Data
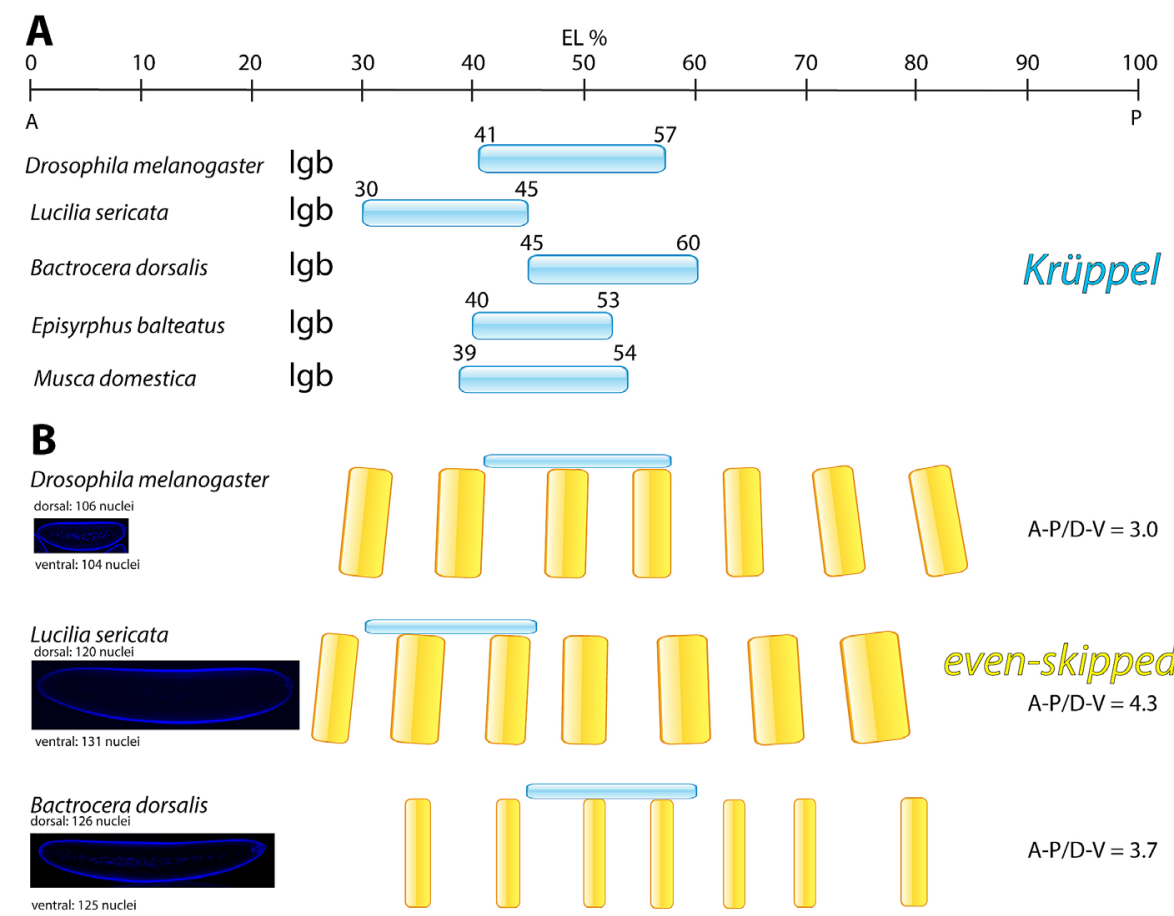

Fig. 7. Schematic representation of relative expression domains of Krüppeland even-skipped in long germband insects. (A) Expression domains in regard to the A-P axis in percentage of egg length (\% EL) of $\mathrm{Kr}$ (blue) in insect families, as reported (Gaul et al., 1987; Rohr et al., 1999, Sommer and Tautz, 1991; Lemke et al., 2010; Blechert et al., 2011) along with those of B. dorsalis. Note the distinct anterior shift in L. sericata (Blechert et al., 2011). Igb indicates "Iong germband insect."(B) Expression domains of even-skipped (yellow) and superimposed Krüppel (blue) in regard to the A-P axis in \% EL in D. melanogaster (Fig. 5M), L. sericata (Fig. 5N) and B. dorsalis (Fig. 5F). Representative nuclear DAPI stainings of embryos are indicated and are shown to scale. The number of nuclei on the dorsal as well as ventral side is indicated. Angles of the bands regarding the A-P axis are indicated in yellow as they appear in the respective insect. The ratio of the $A-P / D-V$ axis is indicated on the right side. Note the prefect perpendicularity of the bands in $\mathrm{B}$. dorsalis with respect to the A-P axis, compared to the other insects. 
from Episyrphus balteatus indicated that the eve stripes were at similar positions as those of Drosophila (Lemke and Schmidt-Ott, 2009), but since the $K r$ band was narrower in comparison to that of Drosophila (Fig. 7A), it did not encompass eve stripe 4 as in Drosophila and consequently, only a single stripe, eve stripe 3 was contained within the $\mathrm{Kr}$ domain.

\section{Evo-Devo: comparative analysis of the shape of the embryos and consequences for the segmentation gene expression at blastoderm stage}

Insects embryos have distinct overall shapes where the primary determinant of the shape is the ratio of the A-P axis versus that of the dorsal-ventral ( $D-V)$ axis. To investigate the cause for this, the number of cells for the A-P axis on the dorsal and ventral side were counted in 3 insect systems, $D$. melanogaster, $L$. sericata and $B$. dorsalis. It should be noted that not only the cell number, but also the size and shape of the individual cells contribute to the overall shape of the insect eggs (Blechert et al., 2011, Mellenthin et al., 2006). For Drosophila, on average $106 \pm 3$ nuclei on the dorsal side versus $104 \pm 3$ nuclei on the ventral side were counted, for Lucilia $120 \pm 4$ nuclei on the dorsal side versus $131 \pm 5$ nuclei on the ventral side, and for Bactrocera $126 \pm 4$ on the dorsal side and $125 \pm 3$ nuclei on the ventral side (Fig. 7B). The ventral side of the Drosophila egg was more rounded, despite the fact that both sides have similar numbers of cells. At close examination, this difference is due to the fact that dorsal cells are highly columnar and the amount of lateral cytoplasm is small, enabling a straight dorsal surface (Fig. 7B). Conversely, ventral cells were slightly constricted at the basal side which enabled a rounded surface. The form of the insect embryo is already determined during the late stages of oogenesis, an observation extended to all long-germband insects. Furthermore, the migration of the nuclei to the periphery during nc 9-10 is a microtubule-driven process not involving changes to the shape of the embryo. Hence, the nuclei of the Drosophila embryo adapted to their environment once they have reached their final position at the cortex. The situation in the Lucilia egg, however, which also shows a rounded ventral surface, is not as pronounced as in Drosophila. Due to its elongated shape, it appears to follow another strategy. The number of nuclei on the ventral side is larger than that of the dorsal side, consequently, leading to almost even spacing on both sides. Bactrocera embryos have similar numbers of nuclei on either side and a slightly rounded ventral side which appears to be controlled in a similar way as in Drosophila.

To address the question whether the different number of cells on the dorsal and ventral side have implications for the expression patterns, a comparison of the angles of the eve stripes with respect to the A-P axis in all 3 insects was conducted. It was noted that the eve stripes in Drosophila and Lucilia were not always perpendicular to the A-P axis, particularly the first and the last stripes. While this behavior can be explained by the curved nature of the ventral side in Drosophila (Fig. 5M), a reasonable explanation could not be given for the elongated Lucilia egg (Fig. 5N). In contrast, a similarly shaped egg such as the Bactrocera egg revealed almost perfectly arranged perpendicular stripes (Fig. 5 D-F). Notably, all $B$. dorsalis segmentation genes analyzed exhibited this behavior at blastoderm stage (Fig. 2-6).

As far as the overall shape of the embryos, there were distinct A-P/D-V ratios between the insects. Drosophila, a relatively compact insect egg showed a ratio of 3.0, while Bactrocera showed a ratio of 3.8 and Lucilia as the largest of the 3 eggs had a ratio of 4.3 (Fig. 7). It is important to note that the ratio has an impact on the mechanical stability of the egg: rounder eggs are more stable than elongated eggs. This fact becomes an issue when the egg is manipulated, e.g. by pricking it with a needle during genetic transformation where Lucilia and Bactrocera are particularly vulnerable, compared to Drosophila (unpublished observations).

\section{Discussion}

We have analyzed the expression of some of the important early A-P axis patterning genes in $B$. dorsalis and found some similarities to known expression patterns in Drosophila, but also some distinct features associated with this oriental fruit fly. Furthermore, we described, in detail, its embryogenesis and imaginal disc development.

We noted some similarities of the appearance of the $B$. dorsalis egg to that of $L$. sericata or $D$. melanogaster. All three insects showed an identical number of segments, including a head, 3 thoracic segments, 8 abdominal segments, and a tail (Fig. 1K). The engrailed gene was an excellent marker for segment number as well as identity, hence, comparison of the en stripe numbers and position might give some clues as to the subdivision of the insect. As evident from Fig. 6, B. dorsalis revealed more en stripes than L. sericata (Mellenthin et al., 2006) or D. melanogaster (Attrill et al., 2016). This is due to a stripe in the head, designated "0" (Fig. 6F) and stripe 16 at the posterior end, while the number and position of the stripes in the trunk seemed constant. These different numbers of stripes may be attributed to different functions of tissues. For example, B. dorsalis females harbor an ovipositor while a true equivalent of this organ is not found in $L$. sericata or D. melanogaster.

It is important to note that in Drosophila, not all cells of the cellular blastoderm contribute to the later larval body structure, referred to as the "fate map of the blastoderm" (Technau and Campos-Ortega, 1985) The precursor cells of T1 (Fig. 1K) were located at about $35 \% \mathrm{EL}$, and $\mathrm{A} 8$ at $\sim 80 \% \mathrm{EL}$, while regions more anterior were precursors for head structures and elements of the internal digestive system such as anterior midgut or esophagus. At the molecular level, parasegments were defined as the metameric units to subdivide the blastoderm embryo, whereby the eve stripes define all odd-numbered parasegments (Martinez-Arias and Lawrence, 1985). In this respect, it is noteworthy that in Drosophila, parasegment 3 which is defined by eve stripe 2 or en stripe 3 corresponds to the anterior half of T1. Hence, neither eve stripe 1 nor en stripes 1 \& 2 contribute to the cuticle pattern, but rather to head structures. Likewise, at the posterior end, eve stripe 7 and en stripe 15 constitute A8. When looking at the relative position of the eve stripes among the 3 different insects, it became evident that the anlagen of the larval cuticle cells were located at distinct regions of the blastoderm (Fig. 7B). In this respect, if the eve stripes serve as a landmark to what extent the blastoderm cells will become progenitors for the exoskeleton of the larva, only about $45 \%$ of the surface of the Bactrocera blastoderm cells (Fig. 6F) will contribute to the exoskeleton which is lower than in Drosophila (56\%) or Lucilia (53\%).

As far as the major toolkit of segmentation genes was concerned, Bactrocera lacked maternal bcd which is a major player on the top of the hierarchy of segmentation genes in many insects. It is a 
generally accepted view that in higher Dipterans, bcdarose through a duplication of an ancestral Hox3/zerknüllt gene. In more basal Diptera where bcd is lacking, it was suggested that the function of bcd is exerted by a maternally - as well as zygotically-expressed Hox3/zerknüllt gene (Stauber et al., 2002). In higher Dipterans, these functions are now separated into two functions exerted by maternally-expressed bcd and a zygotically-expressed zerknüllt gene (Stauber et al., 2002). In short germband insects such as Tribolium where bcd is lacking as well, it was proposed that $h b$ and otd together exert the function of bcd (Schroder, 2003).

Very recently, a report in the midge Chironomus showed that panish, a protein containing a cysteine-clamp DNA-binding motif, can exert similar functions as does bcd in Drosophila (Klomp et al., 2015). Like bcd, panish was strongly expressed at the anterior tip of the fertilized embryo as a result of maternal deposition, and like bcd, formed a mRNAgradient at blastoderm stage. RNAi-mediated knock-down of panish revealed a bicaudal phenotype similar to strong bcdmutations. These results showed that evolution possibly established further systems that enabled patterning the anterior end and that bcd and panish were limited to specific families of flies. Of note, panish was not found in the Bactrocera genome nor was it detectable in two closely-related chironomid species which suggested that panish arose only very recently (Klomp et al., 2015).

The next class of genes in the hierarchy of the segmentation, the gap gene class showed both $h b$ and Krgenes conserved. Compared to Drosophila where the first expression was observed during nc 12 (Knipple et al., 1985; Bender et al., 1988), Bactrocera hb and Kr showed expression from nc 13 on, suggesting that blastoderm identity followed a distinct regulation. During later embryogenesis, however, identical organs were labeled suggesting that the later functions of both genes were retained. The third gap gene, otd, also showed its expression delayed by one nc, compared to Drosophila (Finkelstein and Perrimon, 1990).

Down the hierarchy follows the pair rule gene class (NüssleinVolhard and Wieschaus, 1980) where the eve gene was analyzed. Compared to Drosophila, the number of eve stripes remained conserved, however, it was the evolution of the stripes that was clearly different in the two insects (MacDonald et al., 1986; Fig. 5). Again, transcription of eve in Bactrocera was delayed by one nc, compared to Drosophila (Macdonald et al., 1986). In addition, eye-catching was the fact how these 7 stripes were aligned along the A-P axis between the two insects at cellular blastoderm (Fig. 5F vs. Fig. 5M). These appeared oblique at either end in Drosophila or Lucilia (Fig. $5 \mathrm{M}, \mathrm{N}$ ), while in Bactrocera, all segmentation genes were expressed perpendicular to the A-P axis (Fig. 2-6). We should also bear in mind that the number of cells on the dorsal versus the ventral side was only marginally different in all 3 insects, hence the outcome in the perpendicularity of the stripes must have a different origin.

Our comparative analysis in Fig. 7. demonstrates that there are marked differences across insect families of how the gap genes exert their control on pair-rule genes, evidenced by the position of the $K r$ band along the AP axis in comparison to the eve stripes. eve stripe 2 is a paradigm of how the maternal input, together with the gap genes, control the pair-rule genes (Small et al., 1996). While Bactrocera and Drosophila showed similar overlap of the $K r$ bands with reference to the eve stripes where the anterior border of the $K r$ band was precisely adjacent to the posterior border of eve stripe 2 (Fig. 7B), the position of the Lucilia Kr band was distinct and was moved exactly one eve stripe unit to the anterior. Moreover, eve stripe 4 which in Drosophila and Bactrocera is probably activated by $K r$ (Fig. 7B) does not overlap any longer with the $K r$ band in Lucilia. Likewise, in Episyrphus balteatus, eve stripe 4 does not seem to involve $K r$ regulation either, as the posterior part of the $K r$ band does no longer overlap with eve stripe 4 (Lemke et al., 2010, Lemke and Schmidt-Ott, 2009). From this comparative analysis, we can conclude that regulation of eve stripes 2-4 by $K r$ is not conserved among insect families.

During blastoderm stage at nc 14, we noted a particular behavior for $B$. dorsalis nuclei, as they did not undergo an elongation step as in L. sericata or D. melanogaster. In Drosophila, elongation of the nuclei is initiated by the formation of an inverted bask of microtubules that originate from the centrosomes that are located in the periplasm next to the nuclei (Foe et al., 2000, Foe et al., 1993). These microtubules guide the invagination of a furrow which migrates from the apical to the basal side of the nuclei, until they wrap the nuclei into a cell. The leading edge of the furrow is driven by an actin-myosin interaction that migrates, from the apical to the basal side, along the microtubules, until the cellular membrane encapsules the nucleus fully, thus forming a cell. The elongated form of the cells at cellular blastoderm allow cellular constrictions which are one of the driving forces for gastrulation. In contrast, the $B$. dorsalis cells hardly elongated during nc 14 and instead stayed largely round (Fig. 1D). This was true even for gastrulation which creates difficulties for cells to change the cellular morphology and to assign constrictions that are important for gastrulation. We presume that in Bactrocera, the mechanisms leading to the driving forces for ventral furrow formation or germband extension must be different ones, compared to $D$. melanogaster of $L$. sericata.

During the analysis of segmentation genes in Lucilia (Mellenthin et al., 2006), we noticed that the ratio of the area of naked cuticle to that of the denticle bands varied dramatically when comparing the large Lucilia fly to that of the small Drosophila. In Lucilia, we measured a ratio of $3: 1$, while the one in Drosophila was 1.5:1 (Mellenthin et al., 2006). We also noted that the naked cuticle area was particularly dependent on the patterning activity of the segmentation gene wingless which in Lucilia showed signaling activity over 3 times the distance compared to Drosophila (Mellenthin et al., 2006). Further, using mathematical calculations, it was shown that a 20 -fold increase of the Wingless producing cells still was not enough to compensate for the larger distance between the sender and receiver cell, unless a facilitated movement of the Wingless protein was allowed (Mellenthin et al., 2006). In the case of the Bactrocera cuticle, we noted that the ratio was 2:1 (Fig. 1K) which was closer to that of the small Drosophila, and not in the range of the larger Lucilia. Hence, the ratio of the naked cuticle/denticle belts does not increase linearly with the size of the insect. Rather, these ratios likely represent adaptations of the systems to their habitat or their ability to exert movements. We therefore reasoned that these differences in these ratios should immediately be reflected in the expression patterns of the naked cuticle-patterning genes of these biological systems. In this case, the en gene is good marker gene, as it allows to define several issues important for insect segmentation, this for the following reasons: (i) its anterior expression border defines the parasegmental border, a feature which is conserved across most insect phyla (Mellenthin et al., 2006), (ii) the number of en-expressing cells is a good estimate how large the naked cuticle will be (Mellenthin et al., 2006), (iii) 
the number of en bands is a good marker for estimation of how many segments a fly has (Baumgartner et al., 1987; Mellenthin et al., 2006). When compared to Lucilia where 6 cells were reported to show en expression at extended germband (Mellenthin et al., 2006), Bactrocera en was expressed in a much less wider band, comprising only 2 cells (Fig. 6 F). Hence, the capacity to pattern the cuticle is limited, taken into account the size of the insect. In fact, the much smaller Drosophila embryo also revealed 2 cells expressing en which corroborated the notion that size did not matter.

\section{Materials and Methods}

\section{Maintenance of $B$. dorsalis}

$B$. dorsalis flies were maintained in $25 \times 50 \mathrm{~cm}$ round Plexiglas cages and fed with a constant source providing 3 parts of sugar, 1 part of yeast hydrolysate and water. For larval stages, a mixture $1000 \mathrm{~g}$, sugar, $100 \mathrm{~g}$ yeast hydrolysate, $50 \mathrm{~g}$ yeast extract and $50 \mathrm{~g}$ peptone, supplemented by banana or apple pieces was used. To prevent escape of larvae during the "jumping phase" of larval stages, containers were always covered with a cover during this time.

\section{Embryo collection}

Bactrocera flies were exposed to standard food for 10 days. For embryo collection, a smaller plastic beaker was prepared with many small holes (diameter $>1 \mathrm{~mm}$ ) which was positioned over a freshly-cut apple piece. To enable a precise embryo collection, a pre-collection phase of 2 hours was employed where eggs that were deposited were discarded. In some cases, an apple, cut into a halves, was used for embryo collection, where the embryos were collected by removal of the shell and collecting the embryos from the inner surface of the shell.

\section{Identification of orthologous genes in B. dorsalis}

The Bactrocera orthologues of segmentation genes were identified through standard BLAST searches of a database where access was provided by Scott Geib. This database is accessible via the NCBI Bactrocera dorsalis Annotation Release 100. Note: the maternal bicoid gene is not present in the Bactrocera genome. B. dorsalis hunchback has accession number XM_011208844, orthodenticle XM_011202351.1, KrüppelXM_011207908.1, even-skippedXM_011210455.1 and engrailed XM_011216118.1. hunchback was amplified by PCR primers GCGAAATCACTACAAGATCAG (forw) and TAAATACTTAGGAACGTAACC (rev), orthodenticle with CTCGACAGAAGCCTTAATGGC (forw) and TTATTCGCATTGCCTCCAGCG (rev), Krüppel with ACCCCCATAACCGTGCCGATG (forw) and CTACTCCATTAGGGTGGTTTG (rev), even-skipped with GCGATCAATTGACACGTCTGG (forw) and AAGACTCGGTTTTGTAGGGCT (rev), engrailed with CTCCGCGTTTACTACAACGCC (forw) and GGGACGATCGCTGTAGCGCGT (rev). Each PCR fragment was sequenced to verify its origin.

\section{In situ hybridization}

Templates for Riboprobes were generated using a 0-4 h B. dorsaliscDNA library (L. Ngernsiri, unpublished) as templates and T7 RNA polymerasebinding sites on the reverse primer from the above identified $B$. dorsalis segmentation genes. These DNA templates were purified, sequenced and used as templates using a DIG-labeling kit (Roche) as described (Fahmy et al., 2014). A non-related sense probe was used as a negative control. In situ hybridization followed a protocol according to (Fahmy et al., 2014).

\section{Immunohistochemistry}

D. melanogaster and L. sericata embryos were heat-fixed and stained with a monoclonal antibody against the Even-skipped protein, (mab 2B8, DSHB) at a concentration of 1:250, counterstained with DAPI and monitored on a Zeiss LSM 710 confocal microscope.

\section{Acknowledgements}

S. B. thanks the Swedish Research Council, the Olle Engkvist Byggmästare Foundation, the Kock Foundation and the Eric Philipp Sörensen Foundation for support. We thank Kasetsart University for the Student Exchange Fellowship to W. S. to work at Lund University. We wish to thank Scott Geib for access to his B. dorsalis database prior to publishing their data, Sol Da Rocha for excellent technical assistance and Linda Wei for comments on the manuscript.

\section{References}

ANDERE, A.A., PLATT, R.N., RAY, D.A. and PICARD, C.J. (2016). Genome sequence of Phormia regina Meigen (Diptera: Calliphoridae): implications for medical, veterinary and forensic research. BMC Genomics 17: 842.

ANDERSON, D.T. (1964). The Embryology of Dacus Tryoni (Diptera). 3. Origins of Imaginal Rudiments Other Than the Principal Discs. J Embryol Exp Morphol 12: $65-75$.

ATTRILL, H., FALLS, K., GOODMAN, J.L., MILLBURN, G.H., ANTONAZZO, G., REY, A.J., MARYGOLD, S.J. and FLYBASE, C. (2016). FlyBase: establishing a Gene Group resource for Drosophila melanogaster. Nucleic Acids Res 44: D786-792.

BAUMGARTNER, S., BOPP, D., BURRI, M. and NOLL, M. (1987). Structure of two genes at the gooseberry locus related to the paired gene and their spatial expression during Drosophila embryogenesis. Genes Dev 1: 1247-1267.

BENDER, M., HORIKAMI, S., CRIBBS, D. and KAUFMAN, T.C. (1988). Identification and expression of the gap segmentation gene hunchback in Drosophila melanogaster. Dev Genet 9: 715-732.

BLECHERT, O., DOUGLAS, D. and BAUMGARTNER, S. (2011). Conserved function of the Kruppel gap gene in the blowfly Lucilia sericata, despite anterior shift of expression. Insect Mol Biol 20: 257-265.

BUCHER, G. and KLINGLER, M. (2004). Divergent segmentation mechanism in the short germ insect Tribolium revealed by giant expression and function. Development 131: 1729-1740

CALLA, B. and GEIB, S.M. (2015). MicroRNAs in the oriental fruit fly, Bactrocera dorsalis: extending Drosophilid miRNA conservation to the Tephritidae. BMC Genomics 16: 740

CAMPOS-ORTEGA, J.A. and HARTENSTEIN, V. (1985). The Embryonic Development of Drosophila melanogaster. Springer, Berlin.

CROMBACH, A., WOTTON, K.R., JIMENEZ-GURI, E. and JAEGER, J. (2016). Gap Gene Regulatory Dynamics Evolve along a Genotype Network. Mol Biol Evol 33: 1293-1307.

DAVIS, G.K. and PATEL, N.H. (2002). Short, long, and beyond: molecular and embryological approaches to insect segmentation. Annu Rev Entomol 47: 669-699.

DRIEVER, W. and NÜSSLEIN-VOLHARD, C. (1988). A gradient of bicoid protein in Drosophila embryos. Cell 54: 83-93.

FAHMY, K., AKBER, M., CAI, X., KOUL, A., HAYDER, A. and BAUMGARTNER, S. (2014). alphaTubulin $67 \mathrm{C}$ and Ncd are essential for establishing a cortical microtubular network and formation of the Bicoid mRNA gradient in Drosophila. PLoS One 9: e112053.

FINKELSTEIN, R. and PERRIMON, N. (1990). The orthodenticle gene is regulated by bicoid and torso and specifies Drosophila head development. Nature 346:485-488.

FINKELSTEIN, R., SMOUSE, D., CAPACI, T.M., SPRADLING, A.C. and PERRIMON N. (1990). The orthodenticle gene encodes a novel homeo domain protein involved in the development of the Drosophila nervous system and ocellar visual structures. Genes Dev 4: 1516-1527.

FOE, V.E., FIELD, C.M. and ODELL, G.M. (2000). Microtubules and mitotic cycle phase modulate spatiotemporal distributions of F-actin and myosin II in Drosophila syncytial blastoderm embryos. Development 127: 1767-1787.

FOE, V.E., ODELL, G.M. and EDGAR, B.A. (1993). Mitosis and morphogenesis in the Drosophila embryo: point and counterpoint. The Development of Drosophila melanogaster. (Bate M. and Martinez Arias A., eds.) Cold Spring Harbor Laboratory Press. 1: 149-300.

FRIGERIO, G., BURRI, M., BOPP, D., BAUMGARTNER, S. and NOLL, M. (1986) Structure of the segmentation gene paired and the Drosophila PRD gene set as part of a gene network. Cell 47: 735-746. 
GARCIA-SOLACHE, M., JAEGER, J. and AKAM, M. (2010). Asystematic analysis of the gap gene system in the moth midge Clogmia albipunctata. Dev Bio/344: 306-318.

GAUL, U., SEIFERT, E., SCHUH, R. and JACKLE, H. (1987). Analysis of Kruppel protein distribution during early Drosophila development reveals posttranscriptional regulation. Cell 50: 639-647.

GEIB, S.M., CALLA, B., HALL, B., HOU, S. and MANOUKIS, N.C. (2014). Characterizing the developmental transcriptome of the oriental fruit fly, Bactrocera dorsalis (Diptera: Tephritidae) through comparative genomic analysis with Drosophila melanogaster utilizing modENCODE datasets. BMC Genomics 15: 942.

GILCHRIST, A.S., SHEARMAN, D.C., FROMMER, M., RAPHAEL, K.A., DESHPANDE, N.P., WILKINS, M.R., SHERWIN, W.B. and SVED, J.A. (2014). The draft genome of the pest tephritid fruit fly Bactrocera tryoni: resources for the genomic analysis of hybridising species. BMC Genomics 15: 1153.

GOTO, T., MACDONALD, P. and MANIATIS, T. (1989). Early and late periodic patterns of even skipped expression are controlled by distinct regulatory elements that respond to different spatial cues. Cell 57: 413-422.

HARDING, K., HOEY, T., WARRIOR, R. and LEVINE, M. (1989). Autoregulatory and gap gene response elements of the even-skipped promoter of Drosophila. EMBO J 8: 1205-1212.

HARE, E.E., PETERSON, B.K., IYER, V.N., MEIER, R. and EISEN, M.B. (2008). Sepsid even-skipped enhancers are functionally conserved in Drosophila despite lack of sequence conservation. PLoS Genet 4: e1000106.

ILSLEY, G.R., FISHER, J., APWEILER, R., DE PACE, A.H. and LUSCOMBE, N.M. (2013). Cellular resolution models for even skipped regulation in the entire Drosophila embryo. Elife 2: e00522.

JAECKLE, H., TAUTZ, D., SCHUH, R., SEIFERT, E. and LEHMANN, R. (1986). Crossregulatory interactions among the gap genes of Drosophila. Nature 324:668-670.

JAEGER, J. (2011). The gap gene network. Cell Mol Life Sci 68: 243-274.

JURGENS, G. (1987). Segmental organization of the tail region in the embryo of Drosophila melanogaster. Roux's Arch Dev Biol 196: 141-157.

KLOMP, J., ATHY, D., KWAN, C.W., BLOCH, N.I., SANDMANN, T., LEMKE, S. and SCHMIDT-OTT, U. (2015). A cysteine-clamp gene drives embryo polarity in the midge Chironomus. Science 348: 1040-1042.

KNIPPLE, D.C., SEIFERT, E., ROSENBERG, U.B., PREISS, A. and JACKLE, H. (1985). Spatial and temporal patterns of Kruppel gene expression in early Drosophila embryos. Nature 317: 40-44.

LEBLANC, L., TORA VUETI, E., DREW, R.A.I. and ALLWOOD, A.J. (2012). Host plant records for fruit flies (Diptera: Tephritidae: Dacini) in the Pacific Islands. Proc. Hawaii. Entomol. Soc. 44: 11-53.

LEMKE, S., BUSCH, S.E., ANTONOPOULOS, D.A., MEYER, F., DOMANUS, M.H. and SCHMIDT-OTT, U. (2010). Maternal activation of gap genes in the hover fly Episyrphus. Development 137: 1709-1719.

LEMKE, S. and SCHMIDT-OTT, U. (2009). Evidence for a composite anterior determinant in the hover fly Episyrphus balteatus (Syrphidae), a cyclorrhaphan fly with an anterodorsal serosa anlage. Development 136: 117-127.

LIU, P.Z. and KAUFMAN, T.C. (2004). Kruppel is a gap gene in the intermediate germband insect Oncopeltus fasciatus and is required for development of both blastoderm and germ band-derived segments. Development 131: 4567-4579.

MACDONALD, P.M., INGHAM, P. and STRUHL, G. (1986). Isolation, structure, and expression of even-skipped: a second pair-rule gene of Drosophila containing a homeo box. Cell 47: 721-734

MARTINEZ ARIAS, A. (1993). Development and patterning of the larval epidermis of Drosophila. Cold Spring Harbor Laboratory Press.

MARTINEZ-ARIAS, A. and LAWRENCE, P.A. (1985). Parasegments and compartments in the Drosophila embryo. Nature 313: 639-642.

MAVRAGANI-TSIPIDOU, P. (2002). Genetic and cytogenetic analysis of the olive fruit fly Bactrocera oleae (Diptera: Tephritidae). Genetica 116: 45-57.

MELLENTHIN, K., FAHMY, K., ALI, R.A., HUNDING, A., DA ROCHA, S. and BAUMGARTNER, S. (2006). Wingless signaling in a large insect, the blowfly Lucilia sericata: a beautiful example of evolutionary developmental biology. Dev Dyn 235: 347-360.

MOLINE, M.M., SOUTHERN, C. and BEJSOVEC, A. (1999). Directionality of wingless protein transport influences epidermal patterning in the Drosophila embryo. Development 126: 4375-4384.
NAKAO, H. (2015). Analyses of interactions among pair-rule genes and the gap gene Krüppel in Bombyx segmentation. Dev. Biol. 405: 149-157.

NÜSSLEIN-VOLHARD, C. and WIESCHAUS, E. (1980). Mutations affecting segment number and polarity in Drosophila. Nature 287: 795-801.

OGAUGWU, C.E. and WIMMER, E.A. (2013). Molecular cloning and expression of nanos in the Mediterranean fruit fly, Ceratitis capitata (Diptera: Tephritidae). Gene Expr Patterns 13: 183-188.

OLESNICKY, E.C., BRENT, A.E., TONNES, L., WALKER, M., PULTZ, M.A., LEAF, D. and DESPLAN, C. (2006). A caudal mRNA gradient controls posterior development in the wasp Nasonia. Development 133: 3973-3982.

PEEL, A.D., CHIPMAN, A.D. and AKAM, M. (2005). Arthropod segmentation: beyond the Drosophila paradigm. Nat Rev Genet 6: 905-916.

ROHR, K.B., TAUTZ, D. and SANDER, K. (1999). Segmentation gene expression in the mothmidge Clogmia albipunctata (Diptera, psychodidae) and other primitive dipterans. Dev Genes Evol 209: 145-154.

SCHETELIG, M.F., SCHMID, B.G., ZIMOWSKA, G. and WIMMER, E.A. (2008). Plasticity in mRNA expression and localization of orthodenticle within higher Diptera. Evol Dev 10: 700-704.

SCHRODER, R. (2003). The genes orthodenticle and hunchback substitute for bicoid in the beetle Tribolium. Nature 422: 621-625.

SCOLARI, F., GOMULSKI, L.M., GABRIELI, P., MANNI, M., SAVINI, G., GASPERI, G. and MALACRIDA, A.R. (2014). How functional genomics will impact fruit fly pest control: the example of the Mediterranean fruit fly, Ceratitis capitata. BMC Genet 15 Suppl 2: S11.

SIM, S.B., CALLA, B., HALL, B., DEREGO, T. and GEIB, S.M. (2015). Reconstructing a comprehensive transcriptome assembly of a white-pupal translocated strain of the pest fruit fly Bactrocera cucurbitae. Gigascience 4: 14.

SINGH, S.K., KUMAR, D. AND RAMAMURTHY, V. V. (2010). Biology of Bactrocera (Zeugodacus) tau (Walker) (Dipetera:Tephritidae). Entomological Res. 40:259-263.

SMALL, S., BLAIR, A. and LEVINE, M. (1996). Regulation of two pair-rule stripes by a single enhancer in the Drosophila embryo. Dev Biol 175: 314-324.

SOMMER, R. and TAUTZ, D. (1991). Segmentation gene expression in the housefly Musca domestica. Development 113: 419-430.

SPIROV, A., FAHMY, K., SCHNEIDER, M., FREI, E., NOLL, M. and BAUMGARTNER, S. (2009). Formation of the bicoid morphogen gradient: an mRNAgradient dictates the protein gradient. Development 136: 605-614.

STANOJEVIC, D., SMALL, S. and LEVINE, M. (1991). Regulation of a segmentation stripe by overlapping activators and repressors in the Drosophila embryo. Science 254: 1385-1387.

STAUBER, M., PRELL, A. and SCHMIDT-OTT, U. (2002). A single Hox3 gene with composite bicoid and zerknullt expression characteristics in non-Cyclorrhaphan flies. Proc Natl Acad Sci USA 99: 274-279.

TAUTZ, D. and PFEIFLE, C. (1989). A non-radioactive in situ hybridization method for the localization of specific RNAs in Drosophila embryos reveals translational control of the segmentation gene hunchback. Chromosoma 98: 81-85.

TECHNAU, G.M. and CAMPOS-ORTEGA, J.A. (1985). Fate-mapping in wildtypeDrosophila melanogaster. Wilhelm Roux Arch. Dev. Biol. 194: 196-212.

VARGAS, R.I., PINERO, J.C. and LEBLANC, L. (2015). An Overview of Pest Species of Bactrocera Fruit Flies (Diptera: Tephritidae) and the Integration of Biopesticides with Other Biological Approaches for Their Management with a Focus on the Pacific Region. Insects 6: 297-318.

VARGAS, R.I., WALSH, W., KANEHISA, D., STARK, D. AND NISHIDA, T. (2000) Comparative Demography of Three Hawaiian Fruit Flies (Diptera: Tephritidae) at Alternating Temperatures. Ann. Entomological Soc. Am. 90: 162-180.

WILSON, M.J., HAVLER, M. and DEARDEN, P.K. (2010). Giant, Kruppel, and caudal act as gap genes with extensive roles in patterning the honeybee embryo. Dev Biol 339: 200-211

YONG, H.S., SONG, S.L., LIM, P.E., EAMSOBHANA, P. and SUANA, I.W. (2016). Complete Mitochondrial Genome of Three Bactrocera Fruit Flies of Subgenus Bactrocera (Diptera: Tephritidae) and Their Phylogenetic Implications. PLoS One 11: e0148201

ZHENG, W., LUO, D., WU, F., WANG, J. and ZHANG, H. (2016). RNA sequencing to characterize transcriptional changes of sexual maturation and mating in the female oriental fruit fly Bactrocera dorsalis. BMC Genomics 17: 194. 
Further Related Reading, published previously in the Int. J. Dev. Biol.

Segment formation in Annelids: patterns, processes and evolution

Guillaume Balavoine

Int. J. Dev. Biol. (2014) 58: 469-483

https://doi.org/10.1387/ijdb.140148gb

Gene expression suggests double-segmental and single-segmental patterning mechanisms during posterior segment addition in the beetle Tribolium castaneum

Ralf Janssen

Int. J. Dev. Biol. (2014) 58: 343-347

https://doi.org/10.1387/ijdb.140058rj

Segmentation, metamerism and the Cambrian explosion

Juan Pablo Couso

Int. J. Dev. Biol. (2009) 53: 1305-1316

https://doi.org/10.1387/ijdb.072425jc

Segmenting the fly embryo: logical analysis of the role of the Segment Polarity crossregulatory module

Lucas Sánchez, Claudine Chaouiya and Denis Thieffry

Int. J. Dev. Biol. (2008) 52: 1059-1075

https://doi.org/10.1387/ijdb.072439ls

Segmenting the fly embryo: logical analysis of the role of the Segment Polarity crossregulatory module.

Lucas Sánchez, Claudine Chaouiya and Denis Thieffry.

Int. J. Dev. Biol. (2008) 52: 1059-1075

5 yr ISI Impact Factor $(2013)=2.879$
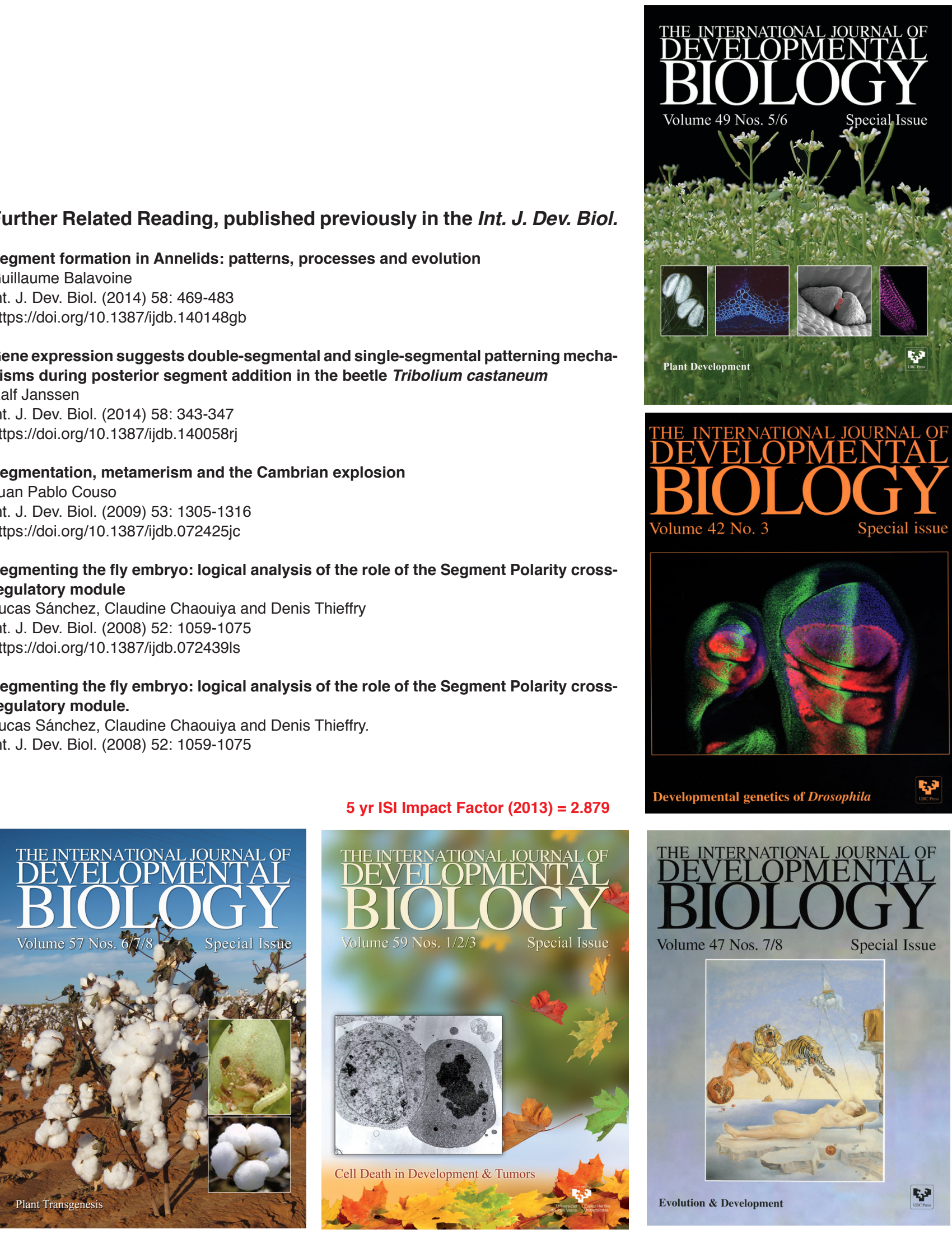

THE INTERNATIONAL JOURNAL OF DEVEIOPNENTA 01007

Volume 42 No. 3

Special issue
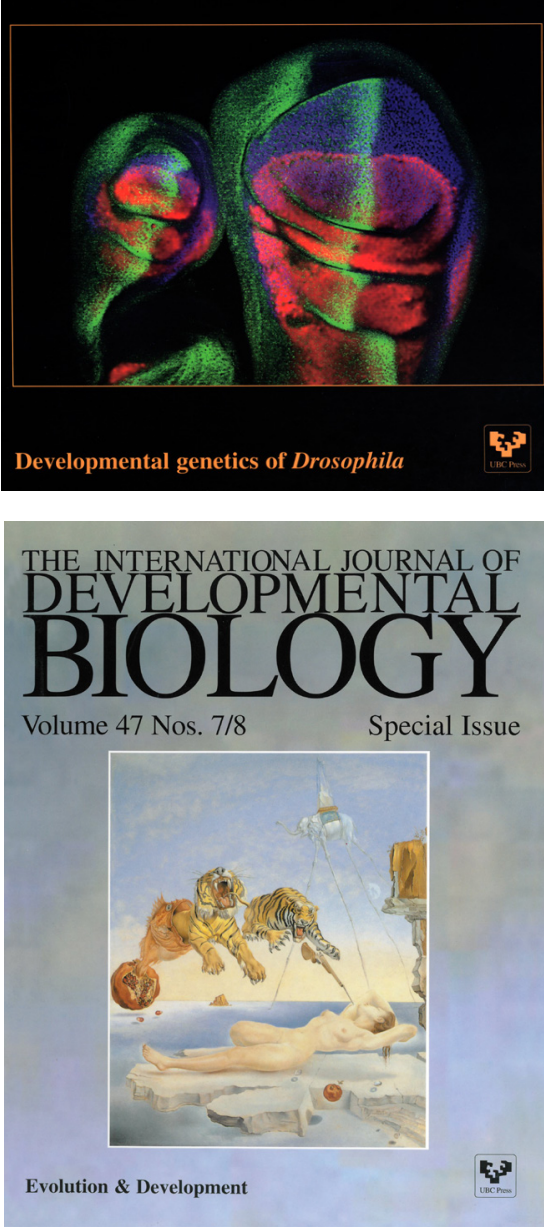